\section{rev Psi}

Revista de Psicología (UNLP)

https://revistas.unlp.edu.ar/revpsi

Artículo de investigación

\title{
Una lectura sobre la diferencia sexual y la época
}

Javier Mariano Pérez ${ }^{1}$

Correspondencia

javiermarianoperez@gmail.com

Filiaciones institucionales

${ }^{1}$ Facultad de Psicología, Universidad Nacional de La Plata(Argentina)
Marisa Badr ${ }^{1}$

\section{Resumen}

Nos encontramos en un tiempo donde muchos sujetos se presentan nombrándose por fuera del llamado binarismo sexual. Un tiempo donde el discurso sobre la diversidad sexual y el género parecen poner en cuestión la noción de diferencia sexual utilizada en psicoanálisis. Desarrollaremos algunos planteos sobre la concepción de la sexualidad que el psicoanálisis freudiano introduce en el discurso de su época, así como algunas referencias lacanianas, para pensar en las incidencias que estos tienen en el discurso actual. Finalmente abordaremos algunos planteos sobre cómo se relacionan la subjetividad, la época y la pulsión. Concluimos con la cuestión de si es necesario pensar un nuevo psicoanálisis o de sostener el desafío al que la clínica una vez más nos convoca.

\section{Palabras clave}

psicoanálisis | diferencia sexual | época actual | pulsión

\section{Cómo citar}

Pérez, J. M. y Badr, M. (2020). Una lectura sobre la diferencia sexual y la época. Revista de Psicología, 19(2), 201-213. doi: 10.24215/2422572XE067

$\begin{array}{ll}\text { Recibido } & \text { Aceptado } \\ 11 \text { may. } 2020 & 18 \text { oct. } 2020 \\ \text { Publicado } & \\ 24 \text { oct. } 2020 & \end{array}$

Claudia E. De Casas (Facultad de Psicología,

UNLP, Argentina) y Nicolás Alessandroni (Facultad de Psicología, UAM, España).
ISSN

2422-572X

Licencia

(c) Copyright: Pérez, J. M. y Badr, M. Licencia de Cultura Libre CC-BY 4.0

Entidad editora

RevPsi es una publicación de la

Facultad de Psicología (Universidad

Nacional de La Plata, Argentina)

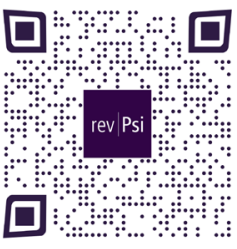

ACCESO ABIERTO

DIAMANTE 


\section{Uma leitura sobre a diferença sexual e a época atual}

\section{Resumo}

Estamos numa época em que muitos sujeitos se apresentam nomeando-se fora do chamado binarismo sexual. Uma época em que o discurso sobre diversidade sexual e gênero parece questionar a noção de diferença sexual usada na psicanálise. Desenvolveremos algumas ideias sobre a concepção da sexualidade que a psicanálise freudiana introduz no discurso de seu tempo, bem como algumas referências lacanianas, para conceituar sobre as incidências que elas têm no discurso atual. Finalmente, abordaremos algumas questões sobre a relação entre a subjetividade, a época atual e a pulsão. Concluímos com a questão de saber se é necessário pensar em uma nova psicanálise ou sustentar o desafio ao qual a clínica mais uma vez nos convoca.

\section{Palavras-chave}

psicanálise | diferença sexual | época atual | pulsão

\section{A perspective on sexual difference and the current times}

\section{Abstract}

We are in a time when many people name themselves outside the so-called sexual binarism. A time when the discourse on sexual diversity and gender seem to question the notion of sexual difference used in psychoanalysis. We will develop some ideas about the conception of sexuality that Freudian psychoanalysis introduces in the discourse of its time, as well as some Lacanian references, in order to conceptualize the incidences that these have in the current discourse. Finally, we will consider some subjects about the relationships between the subjectivity, the current time and the drive. We conclude with the question of whether it is necessary to create a new psychoanalysis or to sustain the challenge to which the clinic once again summons us.

\section{Keywords}

psychoanalysis | sexual difference | current times $\mid$ drive 
Nos encontramos en un tiempo donde muchos sujetos se presentan nombrándose por fuera del llamado binarismo sexual. Ni hombres ni mujeres, ni femeninos ni masculinos. No binarios, andróginos, trans, transgéneros, neutros y otras nominaciones. Estamos en un tiempo donde el discurso sobre la diversidad sexual y el género parecen poner en cuestión la diferencia, al menos como esta es entendida desde el psicoanálisis.

En el año 2010 Norrie May-Welby fue la primera persona declarada de género "neutro". Un australiano que a los cuarenta y ocho años decide operarse y hacer distintos tratamientos para cambiar de sexo, y no consiguiendo identificarse con ninguno, consigue ser reconocido por la Corte Suprema de Australia como de género neutro. En una nota del año 2013, Norrie dice: "Los conceptos de hombre y mujer no me corresponden. La solución más sencilla es no tener ninguna identificación sexual. (...) No me identifico como hombre ni como mujer. Soy femenino/ femenina, y masculino/masculina" (Australia, una sentencia a favor del género neutro en las personas, 2013). En el 2014, la red social Facebook modificó las categorías de género divididas en masculino y femenino, actualmente cuenta con cerca de setenta opciones de género para que ningún usuario se sintiese excluido. Por su parte, la aplicación de citas Tinder ha incluido aproximadamente treinta y siete identidades de género.

Para comenzar a pensar la relación entre sexualidad y época, tomamos una indicación de Lacan (1953/2012), quien plantea:

Mejor pues que renuncie quien no pueda unir a su horizonte la subjetividad de su época. Pues ¿cómo podría hacer de su ser el eje de tantas vidas aquel que no supiese nada de la dialéctica que lo lanza con esas vidas en un movimiento simbólico? (p. 308)

Siguiendo algunos desarrollos de este autor podríamos pensar a la subjetividad de la época en relación al orden simbólico en el que todo sujeto se inscribe, los significantes disponibles en ese orden, que varían con la época, y las significaciones, mandatos e ideales que se desprenden y circulan en torno a ellos. Los sujetos se identificarán a algunos de esos significantes, harán propios ciertos ideales y significaciones que les permitan vivir en su ámbito social; siempre teniendo en cuenta que este proceso para el psicoanálisis comporta una impronta de la contingencia en relación a cuáles significantes son los que lo van a marcar, y la elección inconciente del sujeto en cuanto a qué posición tomó ante ellos. Entre estos significantes e ideales privilegiados que van a marcar al sujeto, se encuentran aquellos referentes a lo masculino y lo femenino.

Es en este punto que retomamos la indicación lacaniana y se abren algunos interrogantes: ¿ Se trata de nuevas presentaciones de la sexualidad? ¿El psicoanálisis está a la altura de la subjetividad de la época? 
Con el fin de intentar dar alguna respuesta a estas preguntas, retomaremos algunos planteos en relación con la concepción de la sexualidad que el surgimiento del psicoanálisis freudiano introduce en el discurso de su época, intentando dar cuenta de los efectos, aún duraderos, que esta concepción ha tenido. En esta línea abordaremos los conceptos de pulsión, la relación con el objeto y la sexualidad infantil, como postulaciones que aún hoy interpelan muchas concepciones establecidas sobre la sexualidad. También veremos algunos enredos y vaivenes teóricos con los que este autor se encuentra a la hora de intentar definir lo femenino y lo masculino a partir de los complejos de Edipo y castración. Continuaremos el recorrido introduciéndonos en algunos aspectos de la teoría de Lacan que creemos que intentan superar algunos de los atolladeros freudianos a partir fundamentalmente de la redefinición del concepto de falo y cuáles son las consecuencias y reparticiones sexuales que se desprenden de esto en dos momentos distintos de su enseñanza, para pensar en las incidencias recíprocas que se producen con el discurso actual. Finalmente, propondremos una lectura de la articulación entre la época, la pulsión y las llamadas nuevas presentaciones de la sexualidad, intentando dar cuenta de qué sería que el psicoanálisis -o un analista- esté a la altura de la subjetividad de la época. Para esto, tendremos en cuenta uno de los aportes fundamentales que creemos que puede hacer el psicoanálisis a la cuestión de la sexualidad en la época actual es el peso teórico y clínico que otorga a la operatoria del Ideal y cuál es la posición que conviene adoptar ante este desde la ética del psicoanálisis.

\section{Freud: la pulsión y la diferencia sexual}

Desde los inicios de la obra freudiana, la sexualidad ocupó un lugar que devino para el psicoanálisis uno de sus pilares fundamentales junto a la hipótesis del inconciente. Es sin duda en su texto Tres ensayos de teoría sexual (Freud, 1905/2010, pp. 123-222) que Freud introduce una concepción de la sexualidad sumamente novedosa para su época. Las formulaciones planteadas allí producen una ruptura tanto con el campo científico como con la opinión popular dominante y los prejuicios de entonces. Esta conceptualización, producto de la clínica freudiana, permitió la ampliación del conocimiento sobre la sexualidad humana hasta entonces limitada a la genitalidad o sexo biológico y la reproducción. Sin duda son numerosos sus aportes, pero nos interesa subrayar algunas consideraciones que permitan retomar cómo ha sido pensada la sexualidad y la diferencia sexual en los inicios del psicoanálisis. Freud se sirve allí no sólo de sus investigaciones respecto de la sexualidad sino también de los aportes del estudio de las perversiones e introduce lo que es uno de los conceptos fundamentales para el campo del psicoanálisis: el concepto de pulsión -Trieb- como pulsión sexual. Su particular apreciación respecto de la indagación del objeto y la meta sexual desviados de la supuesta norma, abrió un cuestionamiento que puso en evidencia, de alguna manera, la falta de un saber sobre el sexo, particularmente en lo referido a la contingencia del objeto de la pulsión, ya que no hay para Freud relación directa entre la pulsión y su objeto. 
La naturaleza de la sexualidad y la inadecuación del ser humano con el objeto de su satisfacción son puestas así en cuestión respecto de lo que hasta entonces se consideraba. Freud distingue la pulsión sexual del instinto y separa así la sexualidad del sexo biológico. En el instinto el objeto está predeterminado, en cambio para la pulsión el objeto es lo más contingente y en la búsqueda de su meta -la satisfacciónla pulsión se sirve de objetos no prefijados. El chupeteo como paradigma de la sexualidad infantil le permite caracterizar a esta última como autoerótica en función de que la satisfacción está en una parte del propio cuerpo.

Freud descubre entonces que lejos de ser un organismo biológico, el cuerpo está afectado por un vivenciar sexual inconciente. La pulsión es definida como un concepto situado entre lo anímico y lo somático,

(...) como un representante [Repräsentant] psíquico de los estímulos que provienen del interior del cuerpo y alcanzan el alma, como una medida de la exigencia de trabajo que es impuesta a lo anímico a consecuencia de su trabazón con lo corporal" (Freud, 1915/2012, p. 117).

Esta conceptualización de la pulsión precisa de alguna manera cómo a lo anímico se le impone un trabajo en función de estar entramado en un cuerpo. Plantea entonces que la pulsión sexual resulta una fuerza que en ocasiones perturba al yo y tiene que luchar contra algunos poderes anímicos que operan como resistencias, entre los que destaca el asco, la moral y la vergüenza. Establece una oposición entre la pulsión sexual y la pulsión de autoconservación para dar cuenta del conflicto que se produce entre la sexualidad y el yo, un yo que en un inicio es entendido como una instancia psíquica desexualizada -concepción que será modificada con la introducción del concepto de narcisismo-.

La organización del campo pulsional de la mano de los complejos de Edipo y castración, y la inscripción de la diferencia sexual con sus consecuencias en lo psíquico, será fundamentada por Freud en la lógica falo/castración, concepción que implica otra ruptura con el campo biológico. En el texto La organización genital infantil (Freud, 1923/2010, pp. 141-149) como un agregado a sus ensayos, no solo reformulará algunas afirmaciones hechas precedentemente, sino que dará cuenta de cómo se produce la organización pulsional a partir del lugar que ocupa el genital masculino para ambos sexos, lo que denominará como el primado del falo. Describirá allí el proceso en el niño, planteando que si bien el pequeño advierte la diferencia entre varones y mujeres no la relaciona inicialmente con lo genital. Partiendo de la premisa fálica de que todo y todos tienen pene, atraviesa un complejo proceso producto de que en sus investigaciones descubre que no es cierta esta suposición, ya que hay quienes no lo tienen, aunque esto no coincide aún con el sexo femenino. Reacciona desconociendo esa falta y construyendo teorías hasta que finalmente llega a la conclusión afectivamente sustantiva de que estuvo y fue removido. Freud dirá: "La falta de pene es entendida como resultado de una castración y ahora se le plantea al niño la tarea de habérselas con la referencia de la castración a su propia persona" (Freud, 1923/2010, p. 147). 
Si bien desarrolla algunas consideraciones del encuentro con la castración en la niña, es interesante subrayar que concluye que el "complejo de castración produce en cada caso efectos en el sentido de su contenido, inhibidores y limitadores de la masculinidad y promotores de la feminidad" (Freud, 1925/2010, p. 275) y que la diferencia entre varón y mujer en cuanto a esta pieza del desarrollo sexual, es entendida como una "comprensible" consecuencia de la diversidad anatómica de los genitales y de la situación psíquica que se enlaza a ella. No se tratará entonces de la diferencia sexual anatómica en sí misma, sino de cómo ésta es interpretada psíquicamente por ambos sexos. Entendemos que Freud hace de la castración una condición ineludible de la subjetividad humana, cuyos procesos según refiere son diversos en los niños y las niñas.

Desde los inicios de su indagación Freud plantea el supuesto de la bisexualidad constitucional del ser humano, por lo que el advenimiento de lo femenino y lo masculino en este sentido no es natural, y articula ambos a la organización pulsional que se produce en los complejos de Edipo y castración. En reiteradas oportunidades señala el carácter impreciso de lo femenino y lo masculino destacando que los individuos humanos, por su disposición bisexual reúnen en sí ambos caracteres, de suerte que "la masculinidad y feminidad puras siguen siendo construcciones teóricas de contenido incierto" (Freud, 1925/2010, p. 276). En algunas ocasiones hace coincidir activo con masculino y pasivo con femenino como avatares del campo pulsional, pero también nos hace saber con la honestidad que lo caracteriza, que no puede esclarecer la esencia de aquello que en sentido convencional o biológico se llama femenino o masculino. Subrayamos una referencia que, creemos, permite situar algo del orden de lo epocal: "Masculino y femenino es la primera diferencia que ustedes hacen cuando se encuentran con otro ser humano y están habituados a establecerla con resuelta certidumbre" (Freud, 1933/2011, p. 105). Como si desde lo perceptivo lo incierto de lo femenino y lo masculino cobrara certeza a través del otro.

Si bien en varias oportunidades parece referir lo masculino y lo femenino a la diferencia sexual en articulación con el atravesamiento del complejo de castración, y su tan mentada "la anatomía es el destino" (Freud, 1924/2010, p. 185), nos encontramos en este recorrido con la dificultad en la que el mismo Freud insiste, en relación al carácter desconocido de lo que constituye la esencia femenina o masculina y que por cierto la anatomía no puede aprehender. En lo incierto algo parece enunciarse como cierto: no depende de la anatomía exclusivamente.

Para el fundador del psicoanálisis, entonces, la diferencia de los sexos no es un dato natural, sino que es el resultado de un complejo proceso, articulado fundamentalmente al recorrido pulsional y a la operatoria de la castración, diferenciándose así de la concepción biológica del cuerpo. La introducción del concepto de pulsión, la consideración de la sexualidad como infantil, parcial y perversa, y el planteo de la bisexualidad estructural del ser humano, constituyen sin duda aportes que subvierten la concepción de la sexualidad humana en tanto se encuentra por fuera de lo que aquella época establecía como norma. 


\section{Lacan: la diferencia sexual a la luz de la teoría del significante y de los modos de goce}

En el anterior apartado hemos visto que la inscripción de la diferencia sexual en Freud dependerá del pasaje por el complejo de castración y las consecuencias psíquicas que tiene la interpretación de la diferencia anatómica, quedando delimitadas dos posiciones inconcientes organizadas en función del binomio fálico/ castrado. Resulta pertinente entonces hacer algunas consideraciones sobre cómo Lacan, en función de su lectura particular del complejo de castración freudiano, reubica y da un estatuto diferente a sus elementos. Nos referimos concretamente a los conceptos de falo y castración. Uno de sus aportes teóricos implica pensarlos a partir de la categoría de lo simbólico, considerando a la castración en este registro no como una fantasía cuyo agente es el padre de la novela edípica, sino como la pérdida de satisfacción inherente a la entrada del sujeto en el lenguaje (Miller, 2005, p. 19). En la misma vía, otros autores afirman que Lacan se diferencia de Freud al postular que la castración es sin padre, no se la puede imputar al padre del Edipo (o a cualquiera de sus personajes) considerado como perturbador del goce, sino que esa pérdida está ya dada de entrada. Es el caso, por ejemplo, de Soler (2009, p. 23), quien afirma que Lacan se separa de Freud respecto al lugar que le da a la castración, ya que para éste la castración está del lado del Edipo, figurada como a causa del padre, mientras que para Lacan queda a cuenta del viviente y su entrada al mundo simbólico.

Podemos postular entonces que de esta lectura de la castración se desprenderá un lugar también diferente del concepto de falo que utiliza Lacan. Si bien pueden encontrarse distintas definiciones en su obra, a los fines de esclarecer las diferencias con la concepción freudiana, nos centraremos en los desarrollos que lo ubican como un significante. Lacan se ocupa de diferenciarlo de una fantasía, de un objeto, e incluso del pene o clítoris que, como significante, puede simbolizar (Lacan, 1958/2011, p. 657). De considerar al falo como significante se desprenden muchas implicancias, pero creemos importante subrayar, de todas ellas, la siguiente: la sexualidad para Lacan será asunto de lenguaje, y como consecuencia de esto, el anhelado acceso al falo siempre se encontrará con una ausencia.

En tanto el significante no remite por sí mismo a un objeto de la realidad, sino que va produciendo un sentido en su articulación con otros, el hecho de que la sexualidad humana esté atravesada por el lenguaje tiene la consecuencia de que "irrealiza las relaciones que han de significarse" (Lacan 1958/2011, p. 661). Entendemos por esto que el lenguaje no describe la realidad como algo que lo preexiste, sino que ésta es fruto de una construcción simbólica, cuyos sentidos dependerán de la articulación de sus elementos. Desde esta perspectiva, entonces, el falo no remitirá a un órgano en particular, sino que su referencia queda alienada por la participación de lo simbólico. Es por esto que está destinado a permanecer velado y siempre que se lo intente objetivar se chocará con una continua evanescencia. 
Esta reubicación conceptual de Lacan hace difícil sostener el binomio que ubica a lo fálico de un lado y lo castrado del otro para designar la repartición sexual, dado que la castración está universalizada en tanto afecta a todo ser hablante, cualquiera sea el genital que tenga.

Cabe remarcar que existen diversos pasajes en la obra freudiana que permiten considerar que el falo no es el pene, sino un lugar simbólico que puede ser encarnado por distintos objetos. Así planteará que el pene es un elemento que puede entrar en una serie sustitutiva a nivel inconciente con otros tales como las heces, el dinero, el regalo, un hijo (Freud, 1917/2009, pp. 113-123; Freud, 1925/2010, pp. 259-276). Es cierto que relacionar el concepto de falo a esta ecuación simbólica no deja de ser una lectura particular que puede realizarse del texto freudiano. Es por esto que consideramos que Lacan aporta claridad a esta diferenciación entre falo y pene, cuestión que, insistimos, en Freud reviste cierta confusión, presentando algunos vaivenes teóricos que tienden a asimilar estos elementos entre sí. Puede decirse que para Freud no son lo mismo, pero su concepción del falo sigue el modelo del pene (Ritvo, 2009, p. 125). Para profundizar esta afirmación es interesante rescatar el planteo de Fischman y Hartman (1995, p. 17), quienes desarrollan tres definiciones del falo en Freud. La primera es la investidura narcisista del pene, que puede ubicarse en el planteo del Edipo en el varón y su salida posibilitada por la renuncia al objeto madre para conservar su narcisismo ante la amenaza de perder el órgano. La segunda definición es el pene del cual la niña se siente privada, en relación a la premisa universal de que todos tienen. La tercera, es que el falo sería el pene que le falta a la madre. Como vemos, estas definiciones marcan una diferencia entre pene y falo, pero no sin tenerlo a aquél como modelo. La concepción que propone Lacan, es inversa, en tanto considera que el pene puede tener una función fálica -así como también otros objetos- de acuerdo al lugar habilitado previamente por el significante.

Hechas las consideraciones sobre estas diferencias entre ambos autores, pasemos a desarrollar cuál es la propuesta lacaniana sobre la relación de los sujetos con el sexo. En un primer momento, este autor continúa la línea de considerar la asunción de la posición sexual en relación a lo fálico exclusivamente. La novedad que introduce respecto de la concepción freudiana es que la diferencia entre los sexos no será pensada solamente en relación al tener sino que incluirá también la dimensión de ser el falo, que definirá a la posición femenina a la altura de La significación del falo (Lacan, 1958/2011, pp. 653-662). En aras de una mayor claridad, sería importante precisar que en la dimensión de la relación entre los sexos de lo que se trataría para los seres hablantes es de un parecer serlo o tenerlo, dado que en tanto significante que designa el objeto del deseo hace que inevitablemente nadie lo tenga ni lo sea.

Lo interesante del planteo de Lacan es que reubica y complejiza los distintos términos freudianos referentes a la inscripción en lo sexual según la teoría del significante, no obstante, el lugar de la mujer sigue quedando estrechamente ligado a la falta. Para el hombre un parecer que protege el tener y para la mujer un parecer que enmascara la falta (Lacan, 1958/2011, pp. 653-662). 
Comenzando la década de 1970, Lacan inaugura otra perspectiva. Según algunos autores como Musachi (2012), esto constituye una respuesta a algunas críticas del feminismo de la época. Es así que los desarrollos que en ese momento incluye en su enseñanza se realizan desde una perspectiva lógica a partir de lo cual, para Lacan, la diferencia sexual será planteada de una manera novedosa, formalizada en las tablas de la sexuación, en donde ubica dos posiciones ya no en función de la anatomía -o para ser más precisos, de la interpretación que los sujetos hagan de ésta- sino en función de un modo de goce de acuerdo a cómo opere la función fálica: todo fálico para el lado hombre, y goce más allá del falo -pero no sin él- para el lado mujer.

Aunque existen diferentes lecturas de la formalización de la sexuación que Lacan realiza en este seminario, intentaremos exponer mínimamente las consecuencias que se desprenden para los sujetos que se inscriben de una y otra manera en relación a la función fálica. En principio es interesante destacar que esta bipartición no implica dos lados opuestos y/o complementarios, en donde los sujetos de un lado encontrarían un objeto de goce del otro lado, sino que en cada lado puede leerse un modo distinto de gozar.

El sujeto que se ubica del lado hombre estaría completamente atravesado por la castración, alguien captado enteramente por el goce fálico, lo que implicaría que el goce sexual se encarrile en la vía del fantasma. Dado que el objeto del deseo como tal está perdido por estructura, Lacan plantea que el fantasma es una forma de sostenerse como deseante: "Digamos que el fantasma, en su uso fundamental, es aquello por lo cual el sujeto se sostiene a nivel de su deseo evanescente, evanescente en la medida en que la satisfacción misma de la demanda le hurta su objeto" (Lacan, 1960, p. 606). La fórmula lacaniana del fantasma implica la relación del sujeto con un objeto, un sujeto que en este fantasma se representa imaginariamente como deseante, afectado por una falta, y localizando en el cuerpo del otro el objeto que colmaría su deseo.

El goce femenino, por otro lado, se plantea como no localizado, no discreto, no fragmentado -al no estar causado por un objeto a- e imposible de ser dicho. Se presenta en manifestaciones del orden de lo ilimitado, desmedido, enigmático e indescifrable. Al no poder universalizarse, e inscribirse en lo simbólico, el lado de lo femenino quedará a cuenta de una por una.

Esta perspectiva de la sexuación no anula necesariamente a la freudiana, pero va más allá, entre otras cosas porque no recurre a lo identificatorio -a la novela edípica- para dar cuenta de lo masculino y lo femenino. Esta propuesta se ubicaría entonces en un más allá del Edipo, dejando de lado las significaciones imaginarias y enfocándose sobre la intersección entre lo real y lo simbólico, en tanto modos de goce con relación al falo y la castración. Esta tiene un lugar como aquello que define la ubicación en una posición o la otra, no desde el plano imaginario, es decir, ordenado en función de quién tiene y quién no, sino en el punto de si el sujeto está o no, habitado por un goce que va más allá de la castración, ilimitado, enigmático, no localizado en un objeto. 


\section{La época y la pulsión}

Del recorrido realizado hasta aquí podemos observar que tanto Freud como Lacan coinciden en dos cuestiones: por un lado, en que no hay ninguna característica psíquica ni anatómica que pueda dar cuenta de lo masculino y lo femenino; y por el otro, en poner de manifiesto la imposibilidad de que en las relaciones entre los seres sexuados pueda existir una armonía o complementariedad, sea cual sea la posición sexual en la que se inscriban. Las distintas formas de nombrarse y ejercer la sexualidad, cada vez más visibles socialmente, nos impulsan a pensar en otros ordenamientos que ofrecen los discursos y prácticas de la época actual que tal vez requieran nuevas categorías conceptuales para abordar este punto. La cuestión que esto pone de relieve es cómo se relacionan los sujetos con los ideales que cada época provee, cómo se ve afectada la pulsión por ellos, y qué mirada tiene -o puede tener- el psicoanálisis frente a esta dinámica.

En el Seminario 2, Lacan plantea que el sujeto que le interesa al psicoanálisis es el sujeto del inconciente, un sujeto que nace en el lenguaje y un inconciente que no podemos pensar sin este. Lacan equipara allí al inconciente con el discurso del otro, postulando:

Este discurso del otro no es el discurso del otro abstracto, del otro en la díada, de mi correspondiente, ni siquiera simplemente de mi esclavo: es el discurso del circuito en el cual estoy integrado. Soy uno de sus eslabones (1954/2008, p. 141).

El lenguaje no sólo produce un sujeto, sino que también crea un cuerpo, en tanto entendemos que la palabra hace acontecimiento en el cuerpo. En esta vía entendemos que la sexualidad, desde los tiempos freudianos y teniendo como referente la conceptualización de la pulsión y sus vicisitudes, se organiza por el lenguaje y no sin resto. El cuerpo no responde a la anatomía sino a las representaciones inconcientes enlazadas a este. Se trata para nosotros de un sujeto del inconciente que, como tal, está atravesado e inmerso en un momento socio-histórico-político, en el discurso de una época determinada que traza sus marcas en las subjetividades. Esto evidencia la relación estrecha entre la época y la pulsión, en tanto entendemos que cada época incide de una manera particular en la vida sexual del parlêtre.

Lacan subraya que "las pulsiones son el eco en el cuerpo del hecho de que hay un decir" (Lacan, 1975/2008, p. 18). La pulsión señala entonces que el cuerpo es sensible al decir. Lo que de la historia de un sujeto se escribe en su cuerpo, deriva en la singularidad de este y es efecto entonces de las marcas del discurso de la época en la que le toca vivir, por estar en relación a los otros que le hablaron.

Si las pulsiones son el resultado de la marca dejada en el organismo por el significante, la animación del cuerpo y su constitución como cuerpo sexuado proviene del encuentro siempre contingente con el otro. Siguiendo este planteo, podemos decir con Lacan que la pulsión, en su pasaje por el Otro como lugar del lenguaje, encuentra algunos significantes que la localizan y dan algunas coordenadas para 
su satisfacción. Masculino y femenino, como todo significante, tienen contenido incierto, y suelen tener la función de inscribir algo de la pulsión, pero no son los únicos. En la época actual existen otros significantes que pueden cumplir esa función, sin necesariamente caer en ese binarismo

Desde la perspectiva del psicoanálisis lo importante en la práctica clínica será ubicar cuáles son los elementos que localizan algo del goce sexual -cuando se han vuelto sintomáticos- estando advertidos de que siempre queda un resto pulsional que no se aviene a ser domesticado por la palabra y los ideales de cualquier época. No obstante, es importante tener en cuenta que por ser psicoanalista no se está exento de guiar la práctica por algunos ideales, aferrándose al horizonte de una época anterior que puede impedir una lectura de lo que aún no se ha escrito en relación a la sexualidad.

Lacan mismo ha denunciado que el psicoanálisis postfreudiano, fundamentalmente el psicoanálisis norteamericano, había sufrido una serie de desvíos que desvirtuaron el sentido originario que Freud transmitió. Para ellos la cura debía estar orientada poniendo el acento en un yo autónomo, donde el paciente debería adaptarse a la realidad e identificarse al analista, constituyéndose este último como un modelo ideal con una elección heterosexual -no está de más aclarar que la IPA prohibía el ingreso a la institución de analistas homosexuales-. Como contraposición a esta orientación, la posición y proposición lacaniana es la de efectuar un retorno a Freud, a sus conceptos fundamentales y a una práctica que no se oriente en sostener un ideal, ni del paciente y mucho menos del analista. Uno de estos conceptos freudianos para pensar en esta proposición es el de Ideal del yo, considerado como una instancia psíquica que intenta recuperar una completud y perfección perdidas. Las perfecciones valiosas que en algún momento habría poseído el yo infantil en su delirio de grandeza, aparecen desplazadas en esta instancia del ideal del yo que en la adultez se erigen como un modelo para el yo (1914/2012, pp. 65-98). La función del ideal entonces es taponar la radical falta de satisfacción de lo pulsional proponiendo formas de hacer con la pulsión que serían sin resto, velando así el vacío estructural que la caracteriza en tanto no existe objeto natural de satisfacción. Creemos entonces que en tanto hay psicoanálisis y psicoanalistas que han tomado la función de promover determinados ideales, la pregunta sobre si el psicoanálisis está a la altura de la época actual podría ser respondida afirmativamente siempre y cuando se opere sosteniendo la distancia entre el Ideal y la pulsión. Siguiendo los desarrollos sobre el concepto lacaniano de deseo del analista que realiza Aramburu (2000), creemos que si abordáramos la clínica sosteniendo un modelo ideal de vivir la pulsión, obturaríamos la singularidad de los sujetos creyendo que existe una buena manera de ejercer la sexualidad y que sería nuestro trabajo velar por ella. Por así decirlo, nos ubicaríamos en una posición prefreudiana. Si nos orientamos en cambio por la ética del psicoanálisis debemos estar dispuestos a escuchar qué modo de vivir la pulsión puede encontrar un sujeto. Se trataría entonces de pensar en los modos singulares de cómo cada sujeto se las arregla con lo sexual yendo más allá de la lógica fálica, aunque no necesariamente prescindiendo de ella. 
Se plantean nuevas presentaciones de la sexualidad contemporánea, fenómenos cada vez más visibles que reclaman un lugar. Nos preguntábamos en el inicio de este trabajo si son nuevas. Podemos decir siguiendo algunas ideas de La Tessa (2016) que no necesariamente se trata en todos los casos de nuevas prácticas sexuales, pero es innegable lo novedoso del modo de la presencia social y política que estas han adquirido en la época actual. Estar a la altura de la subjetividad de la época es la advertencia lacaniana que implica para nosotros los analistas no quedar fijados a paradigmas del psicoanálisis clásico, sino poder escuchar y leer el deseo del sujeto de la subjetividad actual. Se tratará de sostener el desafío al que la clínica una vez más nos convoca produciendo una lectura pertinente. La frase lacaniana que continúa la máxima de estar a la altura de la época refiere: "Que conozca bien la espiral a la que su época lo arrastra en la obra continuada de Babel y que sepa su función de intérprete en la discordia de los lenguajes" (Lacan, 1953/2012, p. 308). Hay discordia en el lenguaje y cada sujeto tiene su propia lengua. Es necesario entonces que el analista se sitúe en su época y sea intérprete de la discordia que el lenguaje produce, ya que allí se encuentra la imposible identidad del ser hablante que las identificaciones no resuelven.

\section{Referencias}

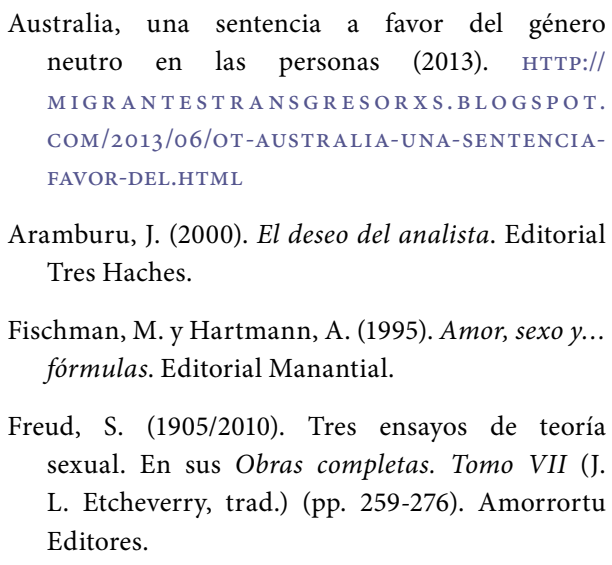

Aramburu, J. (2000). El deseo del analista. Editorial Tres Haches.

Fischman, M. y Hartmann, A. (1995). Amor, sexo y... fórmulas. Editorial Manantial.

Freud, S. (1905/2010). Tres ensayos de teoría sexual. En sus Obras completas. Tomo VII (J. L. Etcheverry, trad.) (pp. 259-276). Amorrortu Editores.

Freud, S. (1914/2012). Introducción del narcisismo. En sus Obras completas. Tomo XIV (J. L. Etcheverry, trad.) (pp. 65-98). Amorrortu Editores.

Freud, S. (1915/2012). Pulsiones y destinos de pulsión. En sus Obras completas. Tomo XIV (J. L. Etcheverry, trad.) (pp. 113-134). Amorrortu Editores.

Freud, S. (1917/2009). Sobre las transposiciones de la pulsión, en particular del erotismo anal. En sus Obras completas. Tomo XVII (J. L. Etcheverry, trad.) (pp. 113-123). Amorrortu Editores.
Freud, S. (1923/2010). La organización genital infantil. En sus Obras completas. Tomo XIX (J. L. Etcheverry, trad.) (pp. 141-149). Amorrortu Editores.

Freud, S. (1924/2010). El sepultamiento del complejo de Edipo. En sus Obras completas. Tomo XIX (J. L. Etcheverry, trad.) (pp. 177-187). Amorrortu Editores.

Freud, S. (1925/2010). Algunas consecuencias psíquicas de la diferencia anatómica entre los sexos. En sus Obras completas. Tomo XIX (J. L. Etcheverry, trad.) (pp. 259-276). Amorrortu Editores.

Freud, S. (1933/2011). 33 a conferencia. La feminidad. En sus Obras completas. Tomo XXII (J. L. Etcheverry, trad.) (pp. 104-125). Amorrortu Editores.

La Tessa, M. (2016). Nuevas presencias de la sexualidad. En La diferencia sexual. Género y psicoanálisis. Letra Viva.

Lacan, J. (1953/2012). Función y campo de la palabra y del lenguaje en psicoanálisis. En Escritos. Tomo I (pp. 231-309). Siglo XXI,

Lacan, J. (1954/2008). El seminario. Libro 2. Ediciones Paidós. 
Lacan, J. (1958/2011). La significación del falo. En Escritos. Tomo II (pp. 653-662). Siglo XXI ediciones.

Lacan, J. (1958/2011). La dirección de la cura y los principios de su poder. En Escritos. Tomo II (pp. 559-615). Siglo XXI ediciones.

Lacan, J. (1975/2008). El seminario. Libro 23. Ediciones Paidós.
Miller, J-A. (2005). Del Edipo a la sexuación. Ediciones Paidós.

Musachi, G. (2012). Mujeres en movimiento. FCE.

Ritvo, J. (2009). El laberinto de la feminidad y el acto analítico. Homo Sapiens Ediciones.

Soler, C. (2009). Lo que usted no podría elegir. Revista Aún, 1, 15-28. 